\title{
QUALIDADE DE FRUTOS DE CAJÁ-MANGA ARMAZENADOS SOB DIFERENTES TEMPERATURAS ${ }^{1}$
}

\author{
DOUGLAS SEIJUM KOHATSU², VALDIR ZUCARELI, WILIAN POLACO BRAMBILLA², \\ REGINA MARTA EVANGELISTA ${ }^{4}$
}

RESUMO - O presente trabalho teve como objetivo determinar o efeito de diferentes temperaturas de armazenamento na qualidade de frutos de cajá-manga. Os frutos de cajá-manga foram obtidos junto ao CEAGESP - Companhia de Entrepostos e Armazéns Gerais de São Paulo, e transportados em caixas isotérmicas para o Laboratório onde foram selecionados quanto à aparência, estádio de maturação, ausência de danos físicos, depois sanitizados em solução de $50 \mathrm{ppm}$ de cloro livre e acondicionados em bandejas de poliestireno expandido, envolto por filme de policloreto de vinila (PVC). O delineamento experimental utilizado foi o inteiramente casualizado, com três tratamentos $\left(4^{\circ} \mathrm{C}, 8^{\circ} \mathrm{C}\right.$ e $\left.25^{\circ} \mathrm{C}\right)$ e 5 épocas de avaliação $(0 ; 3 ; 6 ; 9$ e 12 dias após a instalação do experimento). Em cada coleta, foram avaliadas a firmeza, a acidez titulável, os sólidos solúveis, o teor de ácido ascórbico, a cor da casca e, também, a liberação de $\mathrm{CO}_{2}$ pelos frutos ao longo do tempo. Foi observado que baixas temperaturas prolongam a vida útil dos frutos e que a temperatura de armazenamento influencia nas características avaliadas, sendo a temperatura de $8^{\circ} \mathrm{C}$ a mais adequada para o armazenamento dos frutos de cajá-manga.

Termos para indexação: pós-colheita, frutos tropicais, Spondias dulcis, Anacardiaceae.

\section{FRUIT QUALITY OF CAJÁ-MANGA UNDER DIFFERENT STORANGE TEMPERATURES}

\begin{abstract}
This study aimed to determine the effect of different storage temperatures on fruit quality of ambarella. The ambarella fruits were obtained from the Company CEAGESP-General Warehouses of Sao Paulo and transported in cool boxes to the laboratory, where they were selected for their appearance, maturity and lack of physical damage, then sanitized in $50 \mathrm{ppm}$ chlorine-free solution and packaged in polystyrene trays wrapped with film of polyvinyl chloride (PVC). The experiment was a completely randomized design with three treatments $\left(4,8\right.$ and $\left.25^{\circ} \mathrm{C}\right)$ and 5 times interval $(0,3,6,9$ and 12 days after the experiment installation). In each survey, firmness, titratable acidity, soluble solids, ascorbic acid content and the skin color were evaluated, as well as the release of $\mathrm{CO}_{2}$ by the fruit over time. It was observed that low temperatures prolong the shelf life of fruits and the storage temperature influences the characteristics, and the temperature of $8^{\circ} \mathrm{C}$ was the most suitable for the storage of ambarella fruits.
\end{abstract}

Index terms: postharvest, tropical fruits, Spondias dulcis, Anacardiaceae.

\section{INTRODUÇ̃̃O}

O cajá-manga (Spondias dulcis), fruta exótica originária das Ilhas da Polinésia, é cultivado em pomares domésticos, principalmente no Norte e Nordeste brasileiro. Os frutos são elipsoides, do tipo drupa, com sementes dotadas de fibras rígidas e espinescentes que mergulham parcialmente na polpa. Esta, por sua vez, é suculenta, agridoce e fortemente aromática, sendo muito apreciado in natura (LO-
RENZI et al., 2006).

A vida pós-colheita do cajá-manga é limitada, havendo necessidade de aumentar sua vida útil, reduzir os danos e perdas pós-colheita. Assim, são de fundamental importância o conhecimento e a aplicação de técnicas adequadas capazes de assegurar a manutenção da qualidade dos frutos (SANTOS, 2008).

De acordo com Albuquerque et al. (2010), frutos tropicais podem ter a vida de prateleira prolongada pela redução da taxa respiratória e produção de

\footnotetext{
${ }^{1}$ Trabalho Sinfruit 036 - Simpósio Internacional de Fruticultura - Avanços na Fruticultura (17 a 21 Outubro)

${ }^{2}$ Professores Dr. do Departamento de Ciências Agrárias da Universidade Estadual de Maringá/Umuarama. Estrada da Paca s/n, CEP:87508-10, Umuarama-PR. E-mail: dskohatsu@vem.br

${ }^{3}$ Mestrando do Departamento de Botânica, Instituto de Biociências, Unesp - Botucatu-SP. E-mail: valdirzucareli@yahoo.com.br ${ }^{4}$ Profa, Dra, Departamento de Gestão e Tecnologia Agroindustrial da Faculdade de Ciências Agronômicas da Universidade Estadual Paulista-UNESP. C. postal 237, CEP 18603-970, Botucatu-SP. E-mail: evangelista@fca.unesp.br
} 
etileno, em que técnicas de armazenamentos seriam essenciais neste processo. $\mathrm{O}$ armazenamento refrigerado tem sido amplamente utilizado como método para reduzir perdas pós-colheita.

Por ser um produto de consumo in natura, o emprego da refrigeração prolonga o período de conservação dos frutos, e o uso de atmosfera modificada durante o armazenamento pode reduzir os danos ocasionados pela respiração e pela transpiração, como perda de massa e mudança na aparência (PFAFFENBACHI et al., 2003).

A temperatura ideal de armazenamento, aquela que potencialmente prolongue a conservação pós-colheita sem promover danos fisiológicos nos frutos, é muito variável (NISHIBA; MURATA, 1996). Portanto, o objetivo deste trabalho foi avaliar o efeito de diferentes temperaturas de armazenamento pós-colheita na qualidade de frutos de cajá-manga.

\section{MATERIAL E MÉTODOS}

Foram utilizados frutos de cajá-manga obtidos junto ao CEAGESP- Companhia de Entrepostos e Armazéns Gerais de São Paulo. Após a aquisição dos frutos, eles foram transportados em caixas isotérmicas para o Laboratório de Pós-Colheita de Frutas e Hortaliças do Departamento de Gestão e Tecnologia Agroindustrial da Universidade Estadual Paulista, Câmpus de Botucatu-SP. Os frutos foram selecionados quanto à aparência, estádio de maturação, ausência de danos físicos e, então, sanitizados em solução de 50 ppm de cloro livre.

Após a higienização e classificação, os frutos foram acondicionados em bandejas de poliestireno expandido, envoltos por filme de policloreto de vinila (PVC) e submetidos aos seguintes tratamentos: $\mathrm{T} 1$ - refrigeração a $4^{\circ} \mathrm{C} ; \mathrm{T} 2$ - refrigeração a $8^{\circ} \mathrm{C}$, e $\mathrm{T} 3$ - refrigeração a $25^{\circ} \mathrm{C}$.

$\mathrm{O}$ delineamento experimental utilizado foi o inteiramente casualizado, com 3 tratamentos $(4 ; 8$ e $\left.25^{\circ} \mathrm{C}\right)$ e 5 épocas de avaliação $(0 ; 2 ; 4 ; 6$ e 8 dias após a instalação do experimento). Em cada época, foram avaliadas as seguintes características físico-químicas: textura, acidez titulável, sólidos solúveis, teor de ácido ascórbico, cor e atividade respiratória.

A firmeza dos frutos foi determinada com o auxílio de Texturômetro (STEVENS - LFRA texture analyser), com a distância de penetração de $10 \mathrm{~mm}$ e velocidade de $2,0 \mathrm{~mm} \mathrm{~s}^{-1}$, utilizando-se de ponteira TA 9/1000.

A acidez titulável foi expressa em porcentagem de polpa, obtida por meio da titulação de $5 \mathrm{~g}$ de polpa homogeneizada e diluída para $100 \mathrm{~mL}$ de água destilada, com solução padronizada de hidróxido de sódio a $0,1 \mathrm{~N}$, tendo como indicador fenolftaleína, conforme recomendação do Instituto Adolfo Lutz (BRASIL, 2005).

Os teores de sólidos solúveis (SS) foram determinados na polpa por refratometria, em refratômetro tipo $\mathrm{ABBE}$ (Atago-N1) a $25^{\circ} \mathrm{C}$, conforme as técnicas padronizadas pelo Instituto Adolfo Lutz (BRASIL, 2005), e os resultados foram expressos em ${ }^{\circ}$ Brix.

O teor de ácido ascórbico foi determinado com $10 \mathrm{~g}$ de polpa triturada e diluída em $50 \mathrm{~mL}$ de ácido oxálico a $1 \%$. Após a homogeneização, as amostras foram tituladas com 2,6-diclorofenol indofenol (DCFI) até obtenção de coloração ligeiramente rosada, por 15 segundos. Calculou-se o teor de ácido ascórbico das amostras, tomando-se por base o padrão de ácido ascórbico previamente determinado. Os resultados foram expressos em mg de ácido ascórbico por $100 \mathrm{ml}$ de amostra (BRASIL, 2005).

A determinação da cor foi realizada com leituras em lados opostos de um mesmo fruto, com auxílio de colorímetro Konica Minolta (Choma Meter-400). $\mathrm{O}$ ângulo ${ }^{\circ} \mathrm{Hue}$ representa a rotação numa esfera de cores, em que $0^{\circ}, 90^{\circ}, 180^{\circ}$ e $270^{\circ}$ representam vermelho, amarelo, verde e azul, respectivamente (DING; AHMAD; GHAZALI, 2007).

A caracterização da curva de respiração foi determinada por método titulométrico (BLEINRONTH; ZUCHINI; POMPEO, 1976), e os resultados foram expressos em $\mathrm{ml} \mathrm{CO}_{2} \mathrm{~kg}^{-1} \mathrm{~h}^{-1}$. Para as demais variáveis, os dados obtidos foram submetidos à análise de variância e comparação das médias, utilizando-se do teste de Tukey, ao nível de 5\% de probabilidade.

\section{RESULTADOS E DISCUSSÃO}

A maior taxa respiratória ocorreu no início do experimento, logo após o transporte dos frutos, sendo a incidência de injúrias mecânicas uma possível causa do estresse por trepidação (Figura 1). Durigan, Mattiuz e Durigan (2005) relatam que grande parte das perdas pós-colheita se devem à ocorrência de danos mecânicos durante o transporte. Assim, as injúrias causam danos irreparáveis aos produtos, reduzindo sua vida útil, como resultado do aumento da atividade respiratória e alterações bioquímicas.

Os frutos armazenados a $25^{\circ} \mathrm{C}$ apresentaram taxa respiratória elevada durante todo o período de armazenamento, observando-se, no sexto dia de análise, taxa respiratória 34 vezes maior que nos frutos mantidos a $8^{\circ} \mathrm{C}$. A diferença apresentada durante todo o período de avaliação está de acordo com o descrito por Chitarra e Chitarra (2005), que mencionam que 
a cada $10^{\circ} \mathrm{C}$ de aumento na temperatura duplica-se a taxa respiratória.

No entanto, temperatura inferior ao ideal parece ter aumentado a taxa respiratória, pois frutos armazenados a $4^{\circ} \mathrm{C}$ apresentaram valores superiores àqueles mantidos a $8^{\circ} \mathrm{C}$. Possivelmente, o metabolismo foi acelerado para a manutenção da integridade celular, além de eliminação de metabólitos tóxicos que se acumulam nas células (CHITARRA; CHITARRA, 2005).

$\mathrm{O}$ armazenamento à temperatura de $8^{\circ} \mathrm{C}$ promoveu manutenção da atividade respiratória a partir do segundo dia de análise até o final do experimento (Tabela 1), provavelmente devido à diminuição da taxa respiratória, já que os ácidos orgânicos são substratos para este processo. A relação entre a temperatura e o consumo de ácidos orgânicos pode ser observada nos demais tratamentos, em que os frutos mantidos a $4^{\circ} \mathrm{C}$ apresentaram maior atividade respiratória e consumo de ácidos orgânicos quando comparados com $8^{\circ} \mathrm{C}$. Os frutos armazenados a $25^{\circ} \mathrm{C}$ apresentaram elevada taxa respiratória, atingindo o mínimo conteúdo de ácidos orgânicos já no sexto dia de avaliação. Contudo, houve aumento posterior e pode estar relacionada com o aumento da contaminação microbiológica observado neste tratamento que, consequentemente, pode ter promovido aumento da produção de ácidos, pois os frutos apresentaram odor característico de fermentação.

Para que haja formação de ácidos, os microrganismos utilizam açúcares como substrato, fato que pode estar relacionado ao decréscimo acentuado a partir do sexto dia de avaliação no teor de sólidos solúveis para o tratamento a $25^{\circ} \mathrm{C}$ (Tabela 1). Quando o decréscimo no teor de sólidos solúveis é causado apenas pela utilização dos açúcares no processo respiratório, observa-se redução menos acentuada, assim como apresentado nos frutos mantidos a $4^{\circ} \mathrm{C}$. Este tratamento atingiu as maiores concentrações de sólidos solúveis já no terceiro dia de avaliação, possivelmente devido à aceleração no processo de amadurecimento ocasionado pelo 'chilling'. De acordo com Chitarra e Chitarra (2005), o 'chilling' atua diretamente no processo de produção de etileno por estimular a ACC sintase, enzima responsável por fornecer substrato para a última etapa deste processo.

Os frutos armazenados a $8^{\circ} \mathrm{C}$ apresentaram o máximo teor de sólidos solúveis apenas no último dia de avaliação, mesmo assim não atingiram o mesmo teor dos demais tratamentos, indicando que os mesmos ainda não haviam completado o amadurecimento.

Na firmeza dos frutos, independentemente da temperatura de armazenamento, houve diminuição nos valores desta variável. Porém, esse processo foi mais rápido quando os frutos foram mantidos a $25^{\circ} \mathrm{C}$, o que aconteceu já a partir do terceiro dia de armazenamento, enquanto nos demais tratamentos a redução foi detectada apenas no sexto dia. A firmeza dos frutos armazenados nas temperaturas de $4^{\circ} \mathrm{C} \mathrm{e}$ $8^{\circ} \mathrm{C}$ não diferiu durante todo o período de avaliação.

Os resultados obtidos para a firmeza de frutos estão de acordo com Chitarra e Chitarra (2005), segundo os quais, o aumento da temperatura está associado ao aumento das enzimas responsáveis pela degradação de carboidratos da parede celular, assim como a celulase e a poligalacturonase que desencadeiam o amolecimento dos frutos.

Para a variável teor de ácido ascórbico, foi observada redução no decorrer do armazenamento. Porém, a redução foi mais acentuada nos frutos mantidos a $25^{\circ} \mathrm{C}$ que, ao final do experimento, apresentavam aproximadamente a metade do teor de ácido ascórbico obtido inicialmente.

A redução nos teores de ácido ascórbico observada já no terceiro dia, nos frutos mantidos a $25^{\circ} \mathrm{C}$, demonstra a importância da temperatura de armazenamento não só na qualidade sensorial, mas também na qualidade nutricional de frutos de cajá-manga. $\mathrm{O}$ ácido ascórbico pode ser considerado uma vitamina de fácil degradação, sendo a temperatura um dos fatores que mais influenciam na perda deste composto. Segundo Leme et al. (2007), a estabilidade das vitaminas nos alimentos pode ser afetada por inúmeros fatores, incluindo calor, luz, oxigênio e $\mathrm{pH}$, sendo o ácido ascórbico um dos compostos vitamínicos que se degradam com maior facilidade.

É importante enfatizar o potencial nutricional do cajá-manga para esta vitamina, pois os valores obtidos no presente trabalho estão próximos dos valores relatados por Couto e Canniatti-Brazaca (2010) em laranja 'Pera' (62,5 mg de ácido ascórbico por 100 $\mathrm{ml}$ de polpa).

A coloração dos frutos pode ser interpretada a partir dos dados do ângulo ${ }^{\circ}$ Hue. De acordo com Ramos e Gomide (2007), o ângulo ${ }^{\circ} \mathrm{Hue}$ pode variar de verde $\left(100^{\circ}\right.$ a $\left.200^{\circ}\right)$ a amarelo $\left(70^{\circ}\right.$ a $\left.100^{\circ}\right)$, o que simularia o amadurecimento dos frutos.

Nos frutos armazenados a $25^{\circ} \mathrm{C}$, observou-se mudança de coloração do verde para o amarelo no sexto dia de armazenamento, enquanto os demais tratamentos mantiveram a mesma coloração do início ao fim do experimento. As temperaturas de $4^{\circ}$ e $8^{\circ} \mathrm{C}$ evitaram a degradação de clorofila e diferiram da coloração dos frutos mantidos a $25^{\circ} \mathrm{C}$ já a partir do terceiro dia de armazenamento.

O estudo limitado da influência da temperatura na qualidade pós-colheita de frutos de cajá-manga 
implica a ausência de informações que permitem do presente trabalho permitem elucidar questões discussões mais amplas, e, deste modo, os resultados fisiológicas e bioquímicas durante o armazenamento.

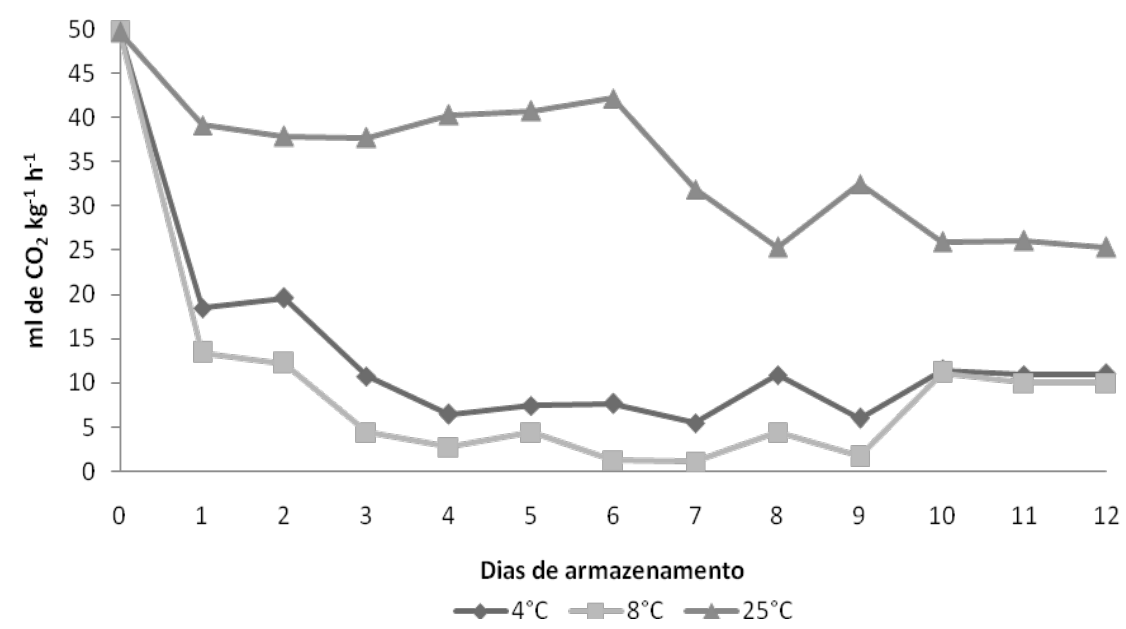

FIGURA 1- Valores médios da liberação de $\mathrm{CO}_{2}\left(\mathrm{ml} \mathrm{CO}_{2} \mathrm{~kg}^{-1} \mathrm{~h}^{-1}\right)$ em frutos de cajá-manga submetidos ao armazenamento sob diferentes temperaturas $\left(4 ; 8\right.$ e $\left.25^{\circ} \mathrm{C}\right)$ durante 12 dias.

TABELA 1- Valores médios de acidez titulável (\%), sólidos solúveis $\left({ }^{\circ}\right.$ Brix), firmeza (g f), ácido ascórbico $\left(\mathrm{mg} 100 \mathrm{ml}^{-1}\right)$ e ângulo ${ }^{\circ} \mathrm{Hue}$ em frutos de cajá-manga armazenados sob diferentes temperaturas, durante 12 dias.

\begin{tabular}{|c|c|c|c|c|c|c|}
\hline \multirow[b]{2}{*}{ Variáveis } & \multirow[b]{2}{*}{ Tratamentos } & \multicolumn{5}{|c|}{ Tempo de armazenamento (dias) } \\
\hline & & $\mathbf{0}$ & 3 & 6 & 9 & 12 \\
\hline \multirow{3}{*}{$\begin{array}{l}\text { Acidez } \\
\text { titulável }\end{array}$} & $\mathrm{T} 1\left(4^{\circ} \mathrm{C}\right)$ & $1,03 \mathrm{aC}$ & $1,19 \mathrm{aAB}$ & $1,22 \mathrm{aA}$ & $1,10 \mathrm{aABC}$ & $1,06 \mathrm{bBC}$ \\
\hline & $\mathrm{T} 2\left(8^{\circ} \mathrm{C}\right)$ & $1,03 \mathrm{aB}$ & $1,15 \mathrm{aAB}$ & $1,11 \mathrm{aAB}$ & $1,20 \mathrm{aA}$ & $1,11 \mathrm{bAB}$ \\
\hline & $\mathrm{T} 3\left(25^{\circ} \mathrm{C}\right)$ & $1,03 \mathrm{aBC}$ & $1,00 \mathrm{bBC}$ & $0,87 \mathrm{bC}$ & $1,14 \mathrm{aAB}$ & $1,27 \mathrm{aA}$ \\
\hline \multicolumn{7}{|l|}{ Valor de F } \\
\hline Trat. x Col. & & & & $8,28 * *$ & & \\
\hline Tratamento & & & & $3,54^{*}$ & & \\
\hline Coleta & & & & $5,47 * *$ & & \\
\hline C.V. $(\%)$ & & & & 7,08 & & \\
\hline \multirow{3}{*}{$\begin{array}{l}\text { Sólidos } \\
\text { solúveis }\end{array}$} & $\mathrm{T} 1\left(4^{\circ} \mathrm{C}\right)$ & $11,63 \mathrm{aB}$ & $14,68 \mathrm{aA}$ & $14,23 \mathrm{abA}$ & $12,03 \mathrm{aB}$ & $11,83 \mathrm{bB}$ \\
\hline & $\mathrm{T} 2\left(8^{\circ} \mathrm{C}\right)$ & $11,63 \mathrm{aB}$ & $12,95 \mathrm{bAB}$ & $12,90 \mathrm{bAB}$ & $12,83 \mathrm{aAB}$ & $13,60 \mathrm{aA}$ \\
\hline & $\mathrm{T} 3\left(25^{\circ} \mathrm{C}\right)$ & $11,63 \mathrm{aBC}$ & $12,43 \mathrm{bB}$ & $15,38 \mathrm{aA}$ & $12,60 \mathrm{aB}$ & $10,68 \mathrm{bC}$ \\
\hline \multicolumn{7}{|l|}{ Valor de F } \\
\hline Trat. x Col. & & & & $8,10 * *$ & & \\
\hline Tratamento & & & & NS & & \\
\hline Coleta & & & & $20,04 * *$ & & \\
\hline C.V. (\%) & & & & 6,24 & & \\
\hline \multirow{3}{*}{ Firmeza } & $\mathrm{T} 1\left(4^{\circ} \mathrm{C}\right)$ & $697 \mathrm{aA}$ & $716 \mathrm{aA}$ & $428 \mathrm{aB}$ & $415 \mathrm{aB}$ & $345 \mathrm{aB}$ \\
\hline & $\mathrm{T} 2\left(8^{\circ} \mathrm{C}\right)$ & $697 \mathrm{aA}$ & $701 \mathrm{aA}$ & $469 \mathrm{aB}$ & $464 \mathrm{aB}$ & $303 \mathrm{aC}$ \\
\hline & $\mathrm{T} 3\left(25^{\circ} \mathrm{C}\right)$ & $697 \mathrm{aA}$ & $320 \mathrm{bB}$ & $201 \mathrm{bBC}$ & $149 \mathrm{bC}$ & $110 \mathrm{bC}$ \\
\hline \multicolumn{7}{|l|}{ Valor de F } \\
\hline Trat. x Col. & & & & $5,33 * *$ & & \\
\hline Tratamento & & & & $64,04 * *$ & & \\
\hline Coleta & & & & $75,07 * *$ & & \\
\hline C.V. (\%) & & & & 16,42 & & \\
\hline
\end{tabular}




\begin{tabular}{|c|c|c|c|c|c|c|}
\hline $\begin{array}{c}\text { Ácido } \\
\text { ascórbico }\end{array}$ & $\begin{array}{l}\text { T1 }\left(4^{\circ} \mathrm{C}\right) \\
\text { T2 }\left(8^{\circ} \mathrm{C}\right) \\
\text { T3 }\left(25^{\circ} \mathrm{C}\right)\end{array}$ & $\begin{array}{l}54,2 \mathrm{aA} \\
54,2 \mathrm{aA} \\
54,2 \mathrm{aA}\end{array}$ & $\begin{array}{l}48,4 \mathrm{aAB} \\
46,2 \mathrm{aA} \\
43,5 \mathrm{aB}\end{array}$ & $\begin{array}{l}41,1 \mathrm{aAB} \\
39,8 \mathrm{aB} \\
30,2 \mathrm{bBC}\end{array}$ & $\begin{array}{l}38,7 \mathrm{aB} \\
37,5 \mathrm{aC} \\
25,5 \mathrm{bCD}\end{array}$ & $\begin{array}{l}35,3 \mathrm{aB} \\
35,9 \mathrm{aC} \\
20,5 \mathrm{bD}\end{array}$ \\
\hline \multicolumn{7}{|l|}{ Valor de F } \\
\hline Trat. x Col. & & & & $16,20 * *$ & & \\
\hline Tratamento & & & & $108,17 * *$ & & \\
\hline Coleta & & & & $60,54 * *$ & & \\
\hline C.V. $(\%)$ & & & & 4,12 & & \\
\hline Ângulo ${ }^{\circ}$ Hue & $\begin{array}{l}\text { T1 }\left(4^{\circ} \mathrm{C}\right) \\
\text { T2 }\left(8^{\circ} \mathrm{C}\right) \\
\text { T3 }\left(25^{\circ} \mathrm{C}\right)\end{array}$ & $\begin{array}{l}110 \mathrm{aA} \\
110 \mathrm{aA} \\
110 \mathrm{~A}\end{array}$ & $\begin{array}{l}108 \mathrm{aA} \\
112 \mathrm{aA} \\
101 \mathrm{bB}\end{array}$ & $\begin{array}{l}106 \mathrm{aA} \\
110 \mathrm{aA} \\
82 \mathrm{bC}\end{array}$ & $\begin{array}{l}105 \mathrm{aA} \\
108 \mathrm{aA} \\
82 \mathrm{bC}\end{array}$ & $\begin{array}{l}104 \mathrm{aA} \\
109 \mathrm{aA} \\
78 \mathrm{bC}\end{array}$ \\
\hline \multicolumn{7}{|l|}{ Valor de F } \\
\hline Trat. x Col. & & & & $14,38 * *$ & & \\
\hline Tratamento & & & & $142,47 * *$ & & \\
\hline Coleta & & & & $25,82 * *$ & & \\
\hline C.V. (\%) & & & & 5,31 & & \\
\hline
\end{tabular}

Médias seguidas de mesmas letras na vertical (minúsculas) e horizontal (maiúsculas) não diferem entre si, pelo teste de Tukey, a 5\% de probabilidade. $*$ : significativo a $5 \%$ de probabilidade. $* *$ : significativo a $1 \%$ de probabilidade. NS: não significativo.

\section{CONCLUSÃO}

A temperatura de armazenamento influência nas características físico-químicas e liberação de $\mathrm{CO}_{2}$ em frutos de cajá-manga, sendo a temperatura de $8^{\circ} \mathrm{C}$ a mais adequada para o armazenamento.

\section{REFERÊNCIAS}

ALBUQUERQUE, E.M.B.; OLIVEIRA, E.N.A.; SANTOS, D.C.; ALMEIDA, F.A.C.; GOMES, J.P. Comportamento dos açúcares redutores em manga in natura armazenada em atmosfera modificada. Tecnologia e Ciência Agropecuária, João Pessoa, v.4, n.3, p.27-31, 2010.

BLEINROTH, E.W.; ZUCHINI, A.G.; POMPEO, RM. Determinação das características mecânicas de variedades de abacate e sua conservação pelo frio. Coletânea ITAL, Campinas, v.7, n.1, p.29-81, 1976.

BRASIL. Agência Nacional de Vigilância Sanitária. Métodos físico-químicos para análise de alimentos. Brasília: Ministério da Saúde, 2005. 1018p.

CHITARRA, M.I.F.; CHITARRA, A.B. Pós-colheita de frutos e hortaliças: fisiologia e manuseio. 2.ed. Lavras: UFLA, 2005. 785p.
COUTO, M.A.L.; CANNIATTI-BRAZACA, S.G. Quantificação de vitamina C e capacidade antioxidante de variedades cítricas. Ciência e Tecnologia Alimentos, Campinas, v.30, n.1, p.15-19, 2010.

DING, P.; AHMAD, S.H.; GHAZALI, H.M. Changes in select quality characteristics of minimally processe carambola (Averrhoa carambola L.) when treated with ascorbic acid. Journal of the Science of Food and Agriculture, London, v.87, p.702-709, 2007.

DURIGAN, M.F.B.; MATTIUZ, B.; DURIGAN, J.F. Injúrias mecânicas na qualidade pós-colheita de lima-ácida 'Tahiti armazenada sob condição ambiente. Revista Brasileira de Fruticultura, Jaboticabal, v.27, n.3, p.369-372, 2005.

LEME, A.C.; GROPPO, V.D.; ROMERO, A.C.; SPOTO, M.H.F.; JACOMINO, A.P. Influência do uso de películas comestíveis em laranja 'Pera' minimamente processada. Boletim do CEPPA, Curitiba, v.25, n.1, p.15-24, 2007.

LORENZI, H.; BACHER, L.; LACERDA, M.; SARTORI, S. Frutas brasileiras e exóticas cultivadas. Nova Odessa: Instituto Plantarum de Estudos da Flora, 2006. 
NISHIBA, I.; MURATA, N. Chilling sensitivity in plants and cyanobacteria: The crucial contribution of membrane lipids. Annual Review of Plant Physiology and Plant Molecular Biology, Palo Alto, v.47, p.541-568, 1996.

PFAFFENBACHI, L.B.; CASTROII, J.V.; CARVALHO, C.R.L.; ROSSETTO C.J. Efeito da atmosfera modificada e da refrigeração na conservação pós-colheita de manga-espada vermelha. Revista Brasileira de Fruticultura, Jaboticabal, v.25, n.3, p.410-413, 2003.
RAMOS, E. D.; GOMIDE, L. A. de M. Avaliação da qualidade de carnes: fundamentos e metodologias. Visçosa: Editora UFV, 2007. 599p.

SANTOS, M.F.G. Qualidade pós-colheita do cajá (Spondias mombin L.) utilizando coberturas de amido de mandioca. In: ANNUAL MEETING OF THE INTERAMERICAN SOCIETY FOR TROPICAL HORTICUlTURE, 54., 2008, Vitória. Proceedings... CD-ROM. 